\title{
Comparison Facilitates Children's Learning of Names for Parts
}

\author{
Dedre Gentner \\ Northwestern University \\ Jeffrey Loewenstein \\ University of Texas \\ Barbara Hung \\ West Roxbury, Massachusetts
}

\begin{abstract}
Learning names for parts of objects can be challenging for children, as it requires overcoming their tendency to name whole objects. We test whether comparing items can facilitate learning names for their parts. Applying the structure-mapping theory of comparison leads to two predictions: (a) young children will find it easier to identify a common part between two very similar items than between two dissimilar items (because the similar pair is easier to align); (b) close alignments potentiate far alignments: children will be better able to extend a novel part name to a dissimilar object after having extended it to a similar object. In three studies, 227 preschool children mapped novel part terms to new animals or objects. Both predictions were confirmed. Children more accurately extended novel part terms to objects that were similar to the standard than to objects that were dissimilar (Experiments 1 and 2), and children more accurately extended novel part names to dissimilar objects after having extended them to similar objects (Experiment 3). We conclude that structure-mapping processes can support part learning.
\end{abstract}

An understanding of part structure is central to the understanding of objects and entities and the mechanisms they participate in (Rosch, Mervis, Gray, Johnson \& Boyes-Braem, 1976; Tversky \& Hemenway, 1984; Tversky, 1989). Yet learning part names might be expected to be quite challenging for children, as it requires them to abandon the whole object level in naming (Markman, 1989) and focus on

Address correspondence to Dedre Gentner, Department of Psychology, 2029 Sheridan Road, Evanston, IL. E-mail: gentner@northwestern.edu 
internal structure. For example, in a test of Markman's whole-object and mutual exclusivity constraints, Markman and Wachtel (1988) presented 3- and 4-year-old children with novel words in the presence of either familiar or novel objects. As predicted, if the objects were unfamiliar (e.g., a lung), children preferred to apply the novel label (e.g., trachea) to the whole object rather than to a specific part of it. However, if the objects were familiar (e.g., a fish) then children would apply a novel label (e.g., dorsal fin) to a specific part rather than to the whole. These findings suggest that, in the absence of another name for an object, children will apply a new word to the whole, not to one of its parts. Consistent with this account, Masur (1997) found that when parents use part terms, they nearly always label a whole object prior to labeling a prominent part. They even appear to use a formulaic sentence structure: "Look, this is an X; it has a Y." (See also Clark, 1993). Both pointing out the name of the whole object and the cuing phrase "has a" appear to facilitate children's acquisition of part labels (Saylor, Sabbagh \& Baldwin, 2002; Saylor \& Sabbagh, 2004).

One way that children can be brought to focus on the parts of an object is for those parts to be used in ongoing actions (Kobayashi, 1997, 1998). Kobayashi (1998) presented 2-year-olds with an unfamiliar object (a u-bolt) with an attached part (a nut). Children who heard a novel label while observing an adult acting on the part (i.e., turning the nut) during familiarization later applied the label to the part and identified the part on a new object (a straight bolt). Children who heard the adult label the part without acting on it did not use or extend the part label. Adult action on a part may facilitate understanding of parts in at least two ways: First, it may allow the child's insight into goals and intentions to inform her interpretation of the part label; and, second, watching the moving part may give rise to a Gestalt "common fate" effect whereby the part's motion as one entity helps to differentiate it from the whole.

Although these factors seem likely to influence part learning, they cannot be the whole story. The mutual exclusivity effect predicts that children should interpret a new word as a part term when they already have a name for the whole. However, this by itself does not tell the child which part is being named. The "moving part" effect has the limitation that it is only applicable to parts that can move relative to the whole object, and requires someone to name the part while it is moving. Indeed in both Markman and Wachtel's (1988) study and Kobayashi's $(1997,1998)$ studies, the experimenters explicitly pointed to the labeled parts. In studies performed by Saylor and Sabbagh, (2004; Saylor et al., 2002), children could only choose to name the color either of the whole object or of one part, thereby removing the need for children to determine which part was being labeled. Such scaffolded learning is not always possible, and may not be necessary. Children can learn words that are not spoken to them (Akhtar, Jipson \& Callanan, 2001; Akhtar, 2005), and can compare across different referents of a word to discover its meaning (Akhtar \& Montague, 1999; Namy \& Gentner, 2002). In addition, no part learning studies 
have addressed the issue of how children apply a part name to a new situation after having been shown the first usage. Our research asks whether children can use comparison processes to map a part word to a new exemplar.

Our basic method was to show children pictures of a novel standard entity (either an object or an animal) along with two alternatives and ask which of the alternatives had the same part: "Look, this one has a fricket. Which one of these also has a fricket?" The alternatives were identical in shape, except that one had the part and the other did not. (See Figure 1). The experimenter never named the entities nor pointed to any specific part of any entity. The dependent measure was whether children could correctly choose which alternative had "a fricket" - that is, which one shared a part with the standard (that was not shared by the other alternative).

We use structure-mapping theory (Gentner, 1983; Gentner \& Markman, 1997) as a framework for discussing the process of aligning and mapping between two entities. A key prediction of structure-mapping is that the process of comparison involves finding a maximal structural alignment between the two representations compared (Falkenhainer, Forbus \& Gentner, 1989; Wolff \& Gentner, 2000). One result of this alignment process is that commonalities are highlighted-that is, corresponding relations and components become more salient. This prediction has been borne out in studies of relational mapping and transfer in adults (Loewenstein, Gentner \& Thompson, 1999; Kurtz, Miao \& Gentner, 2001; Gentner, Loewenstein \& Thompson, 2003) and children (Namy \& Gentner, 2002; Gentner \& Namy, 1999; Loewenstein \& Gentner, 2001, 2005). In our study, children are not given any object names nor any specific actions that identify the parts. Thus, if children are able to correctly identify the alternative with the fricket, this will be evidence that carrying out a structural alignment between the standard and the alternatives-even without knowing what category they belong to-is sufficient to allow children to detect the part in both the standard and the (correct) alternative.

A second prediction of structure-mapping is that there should be strong effects of similarity on whether children can align the standard and the variants, and that these effects should be stronger early in development. This prediction arises, in part, from research showing an experience-driven relational shift in processing of analogy and similarity (Gentner, 1988; Gentner \& Rattermann, 1991; Rattermann $\&$ Gentner, 1998). Early in learning, children (and other domain novices) have rich knowledge of objects but only sparse knowledge of the relations that hold between them. Therefore their perception of similarity, and their ability to carry out structural alignment and arrive at correspondences between two things, relies heavily on the presence of highly similar concrete object matches. With increasing domain knowledge, children's relational representations become deeper and more articulated, making it possible for them to perceive and align purely relational matches (Gentner \& Rattermann, 1991). In the current studies, this predicts that young children will be better able to map the part name to the correct object part, and to iden- 
tify the alternative that shares the part with the standard, if the entities are similar rather than dissimilar.

Experiments 1 and 2 test the above predictions. We showed children a novel standard with a named part and asked whether they could use comparison processes to discern which of two alternatives had a matching part. We varied the similarity of the alternatives to the standard by maintaining (high similarity) or changing (low similarity) the shape of the whole items. The predictions were: (a) despite the lack of direct guidance, children would be able to use comparison to arrive at the correct answer, and (b) as predicted by the relational shift hypothesis, performance would be better with high- than with low-similarity sets.

In the third experiment, we tested a further prediction of structure-mapping theory: that literal similarity (high-similarity) matches will potentiate subsequent analogical matches of lower overall similarity-a progressive alignment effect (Kotovsky \& Gentner, 1996; Gentner \& Medina, 1998). The basis of this claim is that even in close literal similarity matches, comparison processing involves an alignment of relational structure. This alignment raises the salience of the common relational structure, making it easier to notice and map the common structure in a less surface-similar match.

\section{EXPERIMENT 1}

Experiment 1 examined preschoolers' ability to extend new part names to novel objects and animals that were either highly similar (same shape) or highly dissimilar (differently shaped). We examined a fairly broad age range to test whether overall similarity was important only in the early stages, or whether it would continue to facilitate performance even among relatively proficient part-mappers. We showed 3-, 4-, and 5-year-old children a picture of a standard object (an animal or artifact), while labeling one of its parts, and asked them to choose which of two pictured objects shared that part with the standard. All the children received half high-similarity and half low- similarity items, intermixed.

\section{Method}

Participants. Twenty-three 3-year-olds (range: 38-47 months; $M=43.6), 23$ 4-year-olds (range: 48-59 months; $M=54.3$ ), and 25 5-year-olds (range: 60-71 months; $M=66.0$ ) participated through parental responses to newspaper advertisements, mailings, or notices distributed in preschools. Half the children in each age group were female and half were male. No child participated in more than one experiment. 
Materials. The materials were colored drawings, half depicting novel animals and half depicting novel objects. The experimental sets were constructed as follows. First we constructed groups of five figures: a standard that had a given body part and two pairs of variants that were highly similar to the standard. Within each variant pair, one figure had the part and the other did not. For each group we made a sister group of the same type (animal or object), in which the same part was embedded in a very differently shaped animal or object. The parts looked somewhat different in the two sister groups, but were clearly of the same type. Figure 1 shows two examples of such paired groups, one with animals (with wings as the key part) and one with objects (with pockets as the key part). The advantage of having these two paired groups was that high-similarity trials could be produced within a group, and low-similarity trials could be produced by combining the standard from one group with the variants from the other, as shown in Figure 1. Each child received 8 animal trials and 8 object trials, 16 in all: four trials from each of the four cells resulting from crossing item type (animal or object) with similarity type (high similarity and low similarity). The 16 trials were intermixed in semi-random order. Which groups appeared as high or low similarity was counterbalanced across children. Thus, each child saw a given part in only one similarity condition.

The animal parts were hair, tail, nose, arm, wing, belly button, eyebrow, and ear. The object parts were button, door, window, pocket, push button, handle, bow, and open bubble. All parts were internal to the figure, so the presence and absence of parts did not alter the outline of the figure.

Pre-test objects. Each pretest set contained a standard and one pair of test trial items. The two objects were a boat and a lamp. The two parts that were used to illustrate the task were a sail and a lampshade, respectively. These were designed with the goal of making the parts as prominent and obvious as possible. For this reason - and in contrast to the actual test items - the items with and without the parts had different gross shapes, and the standard and matching test item were identical.

Procedure. The experimenter and a participant sat at a table across from each other to play the "parts game." To introduce children to the parts game, they were shown one of the pretest standards (a sailboat or a lamp) and told it had a part (e.g., "This one has a sail"). Children then chose between an identical picture and one without the part (a sailboat without a sail). If children did not choose the picture with the matching part, the experimenter corrected them by directing their attention to the part. The second pretest set was used in the same fashion.

After the pretest sets were presented, the experimenter told children, "We have a bunch of pictures, and they all have new words-and you're going to help me figure out what these words mean." The experimenter introduced a standard and said 

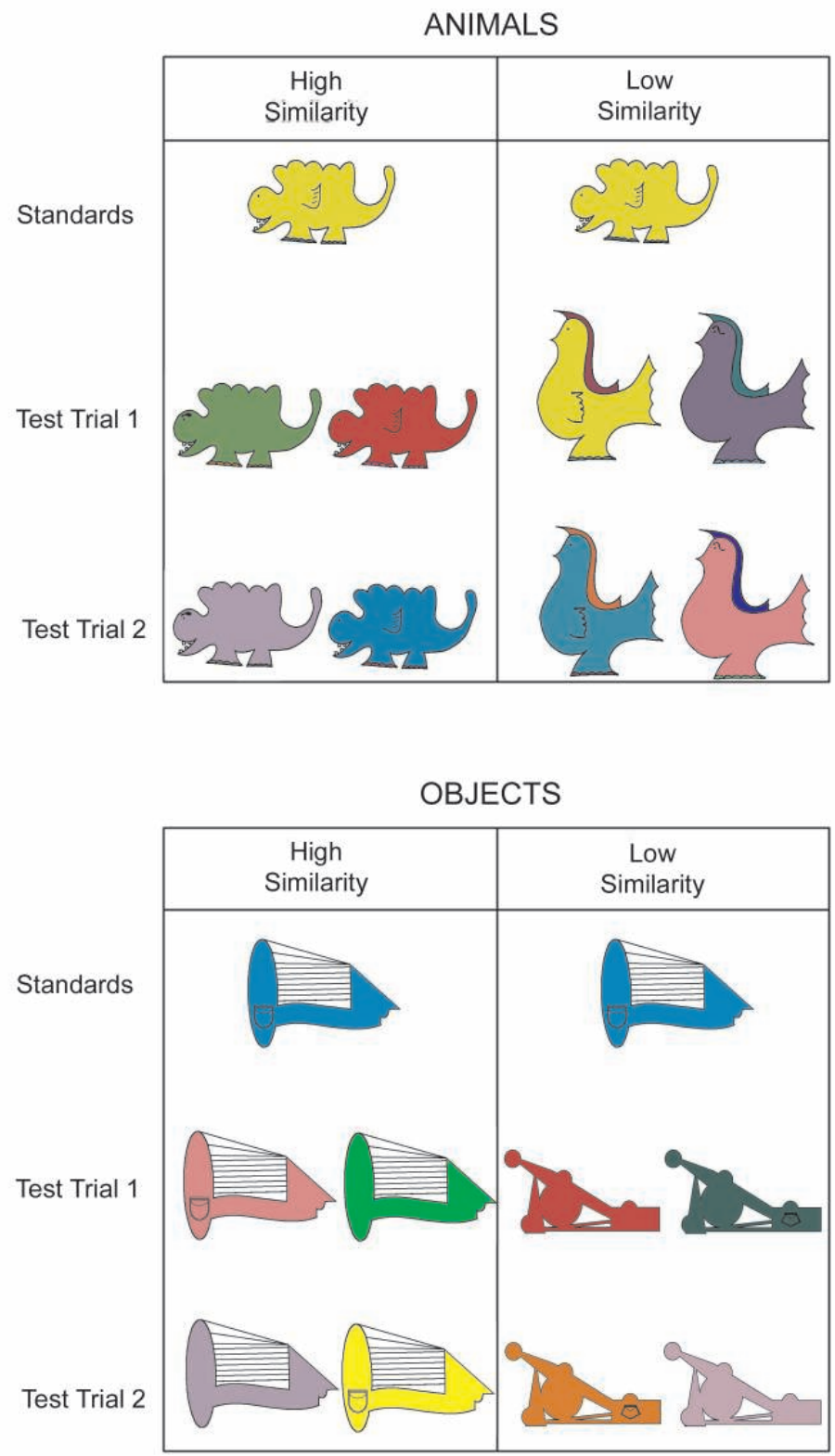

FIGURE 1 Sample animals (both with wings) and objects (both with pockets) from Experiment 1 , showing high- and low-similarity groups. 
(without pointing to the part), "This one/animal has a blick." Then the first pair of test items was presented and the experimenter asked "Which one of these also has a blick?" Once the child made a selection, the experimenter removed the first pair of test variants and presented the second pair, asking, "Which one of these also has a blick?" The standard remained in view at all times. If children pointed to both variants, the experimenter pointed to the standard and said, "We know that this one has a blick and that one of these also has a blick - can you tell me which one that is?" No feedback was given during the experimental trials in this or any other study.

Design and scoring. The data were analyzed in a 3 (Age: $3,4,5) \times 2$ (Similarity: High, Low) $\times 2$ (Type: Animal, Object) mixed measures ANOVA. Similarity and type were within-subjects; age was between-subjects. The order of novel words, the item order, and the position of the correct test item were all counterbalanced. There were no effects of these variables in this study or the subsequent studies.

Children were scored correct on a given set if they were correct on both test trials for that standard. Because children had to choose correctly on both test trials for a given standard, chance performance is $25 \%$. This measure correlates strongly with the simple proportion correct over individual trials $(\min r>.9 ; \min d f=60$ ), and with the proportion correct on the first test trial for each standard $(\min r>.7)$ in this and subsequent studies. Therefore we will report just the set-level measures.

\section{Results}

As predicted, there was an effect of similarity: Children made more correct part-based choices when the test items were similar to the standards (.56) than when they were dissimilar $(.44), F(1,57)=15.83, p<.001, M S E=.05, \eta_{\mathrm{p}}{ }^{2}=.22$ (see Figure 2). Unsurprisingly, older children performed better than younger children (3-year-olds: .34; 4-year-olds: .50; 5-year-olds: .66), resulting in a main effect of age, $F(2,57)=9.57, p<.001, M S E=.21, \eta_{\mathrm{p}}{ }^{2}=.25$. Finally, children performed better on the object sets than on the animal sets (.59 vs. .41, respectively), $F(1,57)$ $=38.24, p<.001, M S E=.05, \eta_{\mathrm{p}}^{2}=.40$.

The specific age group contrasts were consistent with the broader patterns (see Figure 2). In broad outlines, children performed better on objects than on animals, and better on high-similarity than on low-similarity trials. The 3-year-olds performed at chance levels (.25) for the animals and the low-similarity objects. They performed reliably above chance on the high-similarity objects, $t(19)=4.59, p<$ .001 , and significantly better on these than on the low-similarity objects, $t(19)=$ $2.40, p<.05$. The 4-year-olds performed above chance on the high- but not the low-similarity animals, $t(19)=3.05, p<.01, t(19)=1.20, p=.20$, respectively, and showed a significant performance advantage for high- over low-similarity animals, $t(19)=2.18, p<.05$. They performed above chance, and comparably well, 
Objects

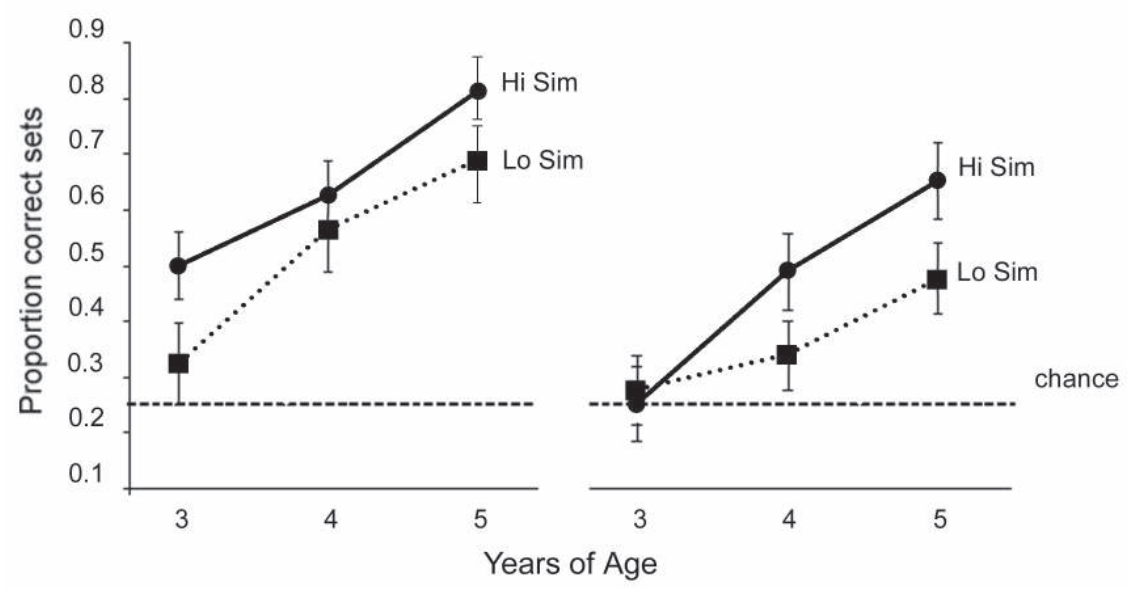

FIGURE 2 Performance on objects and animals by age and similarity for Experiment 1 .

on the high- and low-similarity objects. The 5-year-olds performed reliably above chance on all types. They performed reliably better on high- than on low-similarity animals, $t(19)=3.04, p<.01$, but the similarity advantage on the objects did not reach significance, $t(19)=1.95, p=.07$. Across ages, more individual children performed reliably above chance on high-similarity animals (10/60) than on low-similarity animals $(1 / 60), \chi^{2}=8.11, p<.01$. There was no difference for the objects: $M($ high-sim $)=17 / 60$ and $M($ low-sim $)=16 / 60$.

\section{Discussion}

The results of this study are consistent with the predictions that comparison promotes the detection of common structure. When children were given highsimilarity triads, which are readily alignable, they were better able to identify the common part than if they saw low-similarity triads in which alignment is more difficult. Many of the advantages children have been given in prior experiments on part learning have been stripped away in this task. The experimenter neither pointed to nor demonstrated the part, as was done in Kobayashi's $(1997,1998)$ studies. Children were not given a name for the standard, and hence could not use mutual exclusivity to infer that the new word named only part of the item, and not the whole item. Finally, the items themselves were novel shapes, not members of familiar basic-level categories as in Mintz and Gleitman's (2002) studies. Yet de- 
spite all these hurdles, by four years of age, children were able to carry out a comparison and correctly infer which variant had the corresponding part.

The children had two kinds of support: First, the familiar part-whole phrase "It has a __," guides children to seek an object part (Saylor \& Sabbagh, 2004; Saylor, et al., 2002); and, second, they had a ready comparison from which they could derive new information by aligning the structure of the standard with that of the alternatives. As predicted by structure-mapping, the alignment process is easier when the two figures are perceptually similar. Children more readily discerned which new figure had the same part as the standard when the alternatives were highly alignable with the standard. (Note that this similarity advantage is not simply a preference for choosing a high-similarity item over a low-similarity item. Rather, children are more accurate at choosing between two items which are both highly similar to the standard than between two items less similar to the standard.) The pattern of results suggests that concrete overall similarity facilitates children's ability to align two representations, and therefore their ability to discover a corresponding novel part.

Children's superior performance on objects over animals was unexpected; further inspection suggests that this resulted from specific properties of the stimuli that made the object parts easier to identify than the animal parts. Whereas the animals all had at least one alternative internal part (e.g., eyes and noses along with the novel part), the objects had no other internal parts. The number of alternative internal parts was strongly correlated with children's overall performance for each part, $r(16)=.71, p<.005$. In addition, the object parts were all closed forms, whereas most of the animal parts were open curves, such as a wing or an eyebrow. (However, the correlation between children's part performance and whether the form was closed did not reach significance, $r_{s}=.46, p=.07$.)

Part similarity. To test whether the object advantage could have stemmed from greater part similarity, we asked 10 adult participants to rate the similarity of all the low-similarity pairs. Although the object part pairs $(M=3.92)$ were more similar to each other than the animal part pairs, $(M=3.13), t(9)=3.87, p<.001)$, there was no correlation between these similarity ratings and children's performance, $r(16)=.001, p=.997$.

Part familiarity. To discover whether part familiarity influenced children's performance, we asked an additional group of 114 -year-olds to name the key parts in the 16 standards. There was no relationship between their naming success and the experimental group's performance on the part-mapping task, $r(16)=-.18$, n.s. Every child showed more familiarity with the animal parts than with the object parts (the overall naming means were .76 versus .41 correct) — the opposite pattern of performance from the part-mapping task. Thus, part familiarity does not seem a viable explanation for children's performance. 
Category naming. Although we did not name the objects, children could have assigned their own names and used these to disambiguate the part names. To gauge the likelihood of this, we asked a new group of 13 3- and 4-year-olds to name all 32 possible standards. Children responded, "Don't know" about $40 \%$ of the time, with no significant difference between animals and objects. Further, the names produced were quite variable; across children there was a mean of 4.5 names for each animal and 4 for each object. More importantly, there was no correlation between the proportion of "Don't know" responses and proportion correct on the part-mapping task for any age group, nor for all ages combined. Indeed, a median split on the proportion of "Don't know" responses showed a nonsignificant advantage for the less namable items in the part-mapping task. Thus, we conclude that categorizing the standards is not necessary for success in this task.

The results so far offer encouragement for the claim that children can identify a part and extend it to another item by aligning the standard with the variants. However, the methods of Experiment 1 leave open a possible alternative explanation. For each pair of alternatives, one of the two variants had a part that matched the standards; the other variant was identical except that it lacked that part. Thus the task could have been done by noticing the presence of a part in one variant and the absence of a part in the other, rather than by structurally aligning the variants with the standard. For example, children could simply have chosen the more complex test item. This seems unlikely, because such a strategy would not have produced the observed advantage for high-similar trials. Nonetheless, to ensure that the task requires an alignment with the standard, we conducted another study in which each of the two variants had a unique novel part, only one of which matched the standard's part.

\section{EXPERIMENT 2}

In Experiment 2, children were given the same basic task and materials as in Experiment 1 , except that we added an extraneous part to the incorrect item on each trial. Thus, children had to identify the variant with a matching part, as opposed to simply identifying the variant with an extra part. The predictions of structure-mapping remain the same: children should discern the matching part more accurately when the variants are similar to the standard than when they are different.

There were three other changes in methodology. First, similarity was varied between-subjects instead of within-subjects as in Experiment 1, in order to be sure that the similarly advantage did not depend on a contrast effect. Second, to reduce the number of trials, we used only the eight animal sets. The animal sets were both more challenging and more interesting to the children than the object sets. It also seemed that the animal parts, more clearly than the object parts, derived their meaning from the structure of the whole figure. Finally, we only tested 3- and 4-year-old children, as these ages were sufficient to examine the patterns of interest. 


\section{Method}

Participants. Participants were 87 3- and 4-year-olds who participated at their preschool. (One additional child was not included because of failure to complete the task.) The 3-year-olds ranged in age from 37 to 47 months $(M=42)$, with 20 in the low-similarity condition and 23 in the high-similarity condition. The 4-year-olds ranged in age from 48 to 58 months $(M=52)$, with 23 in the low-similarity condition and 21 in the high-similarity condition. There were approximately the same number of boys and girls in each age and condition.

Stimuli. There were eight stimulus sets, all pictures of animals, modified from their use in Experiment 1. Each set included one standard and two pairs of variants, and each set used a different part. The high-similarity and low-similarity sets were designed as in Experiment 1. The one difference was that whereas in Experiment 1, the incorrect choice in each variant pair simply lacked the correct part, in Experiment 2 the incorrect choice not only lacked the matching part but had an extraneous, non-matching part, as shown in Figure 3. The parts (and their non-matching foils) were wing (with an eyebrow as a foil), nose (hair), eyebrow (horns), hair (belly button), arm (hair), belly button (beard), tail (wing), and ears (mustache). Each child was tested on all eight parts, one on each of the eight sets.

Procedure. Participants were run at their preschool. They were seated in a quiet room, at a table across from the experimenter. Children received the same directions as in Experiment 1 and the same pre-training trials. The basic part-name

\section{Standard}

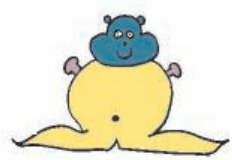

High-similarity variants

Low-similarity variants
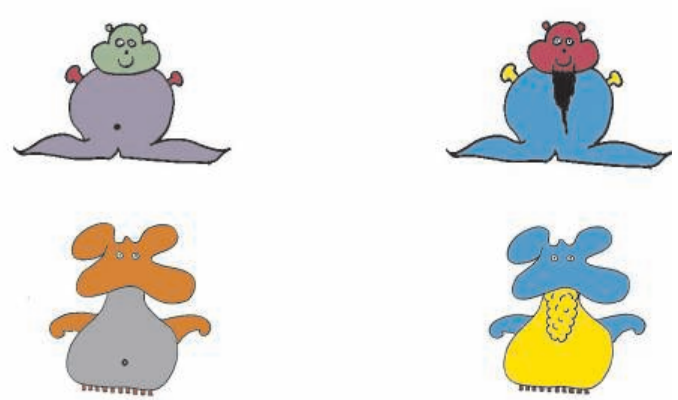

FIGURE 3 Sample items for Experiment 2, showing alternatives for both high-similarity and low-similarity trials. 
extension task was as in Experiment 1, except that children in this study were assigned either to the high-similarity condition or the low-similarity condition, and saw either all high-similarity or all low-similarity sets.

Design and scoring. The data for all the trials were analyzed in a 2 (Age: 3 , 4) $\times 2$ (Similarity: High, Low) ANOVA, with both factors between-subjects. The dependent measure was children's proportion correct on the set measure (that is, two correct choices for a given standard).

\section{Results}

As before, we found the predicted effect of similarity (Figure 4). Children in the high-similarity condition (.40) were better able to identify the matching part than were children in the low-similarity condition $(.30), F(1,83)=4.75, p<.05, M S E=$ $0.05, \mathrm{p}^{2}=.05$. There was also a main effect of age: 4 -year-olds were more accurate than 3 -year-olds (.41 vs. .30), $F(1,83)=5.24, p<.05, \eta_{\mathrm{p}}^{2}=.06$. There was no interaction between age and similarity condition.

Children in the high-similarity condition (.40) performed reliably above chance (.25), but those in the low-similarity condition (.30) did not, $t(43)=3.74, p<.005$, $t(42)=1.83, p=.07$, respectively. Among 3 -year-olds, the low-similarity group

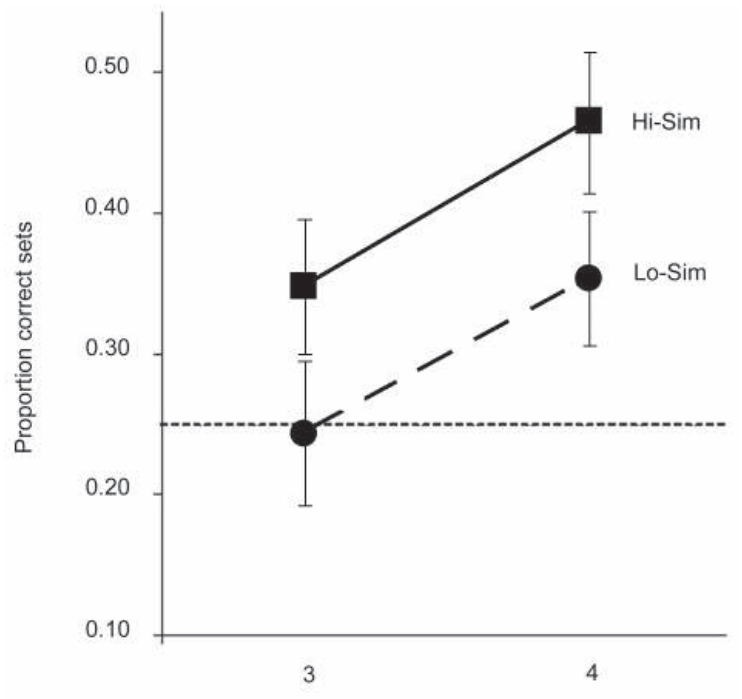

Years of Age

FIGURE 4 Performance in Experiment 2 by age and similarity. 
performed at chance, and the high-similarity group was only marginally different from chance, $t(22)=2.02, p=.06$. Among 4-year-olds, both the low- and high-similarity groups performed above chance, $t(22)=2.37, p<.05, t(20)=$ $3.23, p<.005$, respectively.

We also analyzed the proportion of individual children who performed above chance- that is, those who were correct on at least 5 of the 8 sets (using a binomial distribution with chance at 25\%). Among the 3-year-olds, none of the lowsimilarity group met criterion, but $17 \%$ of the high-similarity group did. Among the 4-year-olds, $22 \%$ in the low-similarity group and 38\% of the children in the high-similarity group met criterion, a nonsignificant difference, $\chi^{2}=1.41, p=.25$. Collapsing to increase sample size, the age difference was reliable, $\chi^{2}=5.67, p<$ .05 , and the condition difference was not, $\chi^{2}=3.39, p=.07$.

\section{Discussion}

The results again showed that 4-year-olds can use comparison processes to map a novel body part from a novel standard to another novel figure. This effect held even in the presence of a mismatched part in the foils. These results suggest that comparison processing can serve as an effective learning mechanism. The findings also bear out the predictions of structure-mapping theory, both in the power of the structural alignment process to inform and in the early reliance on concrete similarity in carrying out an alignment. In the next study we turn to the question of whether structural alignment can help children in another way: Can children learn something from easy concrete alignments that carries forth into more difficult, less concrete matches?

\section{EXPERIMENT 3}

We have found that children can use comparison processes to foster understanding of part labels, and that for young children, the comparison process is more reliable when there is strong overall similarity to constrain the alignment. Next we ask whether comparing highly similar items facilitates identifying the part across low-similarity items. Although it is possible that children in Experiments 1 and 2 chose based on the basis of an implicit global similarity rather than specific awareness of the part similarity, a transfer effect would lend greater support to the claim that children are identifying parts. The idea is that carrying out the high-similarity alignment helps children gain insight about the part and its relation to the whole. Children may therefore be better able to align the low-similarity figures and discover the corresponding part. This prediction derives from a process Kotovsky and Gentner (1996) called progressive alignment: the extraction of a relatively abstract relational structure by making a series of (at least initially) high-similarity compar- 
isons. The striking point about this claim is that comparing pairs that are strongly overall similar is not a dead end, but actually facilitates seeing the match between fairly dissimilar pairs that share the same underlying relational structure.

There is some empirical support for progressive alignment. Kotovsky and Gentner (1996) gave children a perceptual choice task and found that after aligning several closely similar items (such as big/little/big circles with big/little/big squares), 4-year-olds were better able to detect a more abstract relational match: That is, a match between like patterns across different dimensions (e.g., big/little/big circles and dark/light/dark squares) than children who lacked experience with the close similarity items.

There is also evidence that progressive alignment operates in word extension tasks. Waxman and Klibanoff (2000; Klibanoff \& Waxman, 2000) found that preschool children who first extended novel adjectives from a standard to highly similar items subsequently performed better than a baseline group at extending the adjectives to less similar items from a different category. Gentner and Namy (1999, 2002) found that 4-year-olds who were given two highly similar standards (such as an apple and an orange) and encouraged to compare them were likely to extend a new noun label according to taxonomic category instead of to perceptual appearance (e.g., choosing a banana instead of a round balloon); in contrast, children who heard the new label applied to only one of the standards (just the orange) generally chose the perceptual match (the balloon).

To examine more fully whether progressive alignment could be a factor in children's learning of part names, in Experiment 3 we gave half the children (the progressive alignment group) two sets with variants highly similar to the standard followed by two sets with dissimilar variants. The other half of the children (the control group) received dissimilar variants throughout. The last two sets, with dissimilar variants, were the same for both groups.

The dissimilar variants were always from the same group, so children in the control condition received twice as much experience with these kinds of lowsimilarity comparisons. One might therefore predict that children receiving four examples of the same kind of low-similarity comparison would perform better on the last two trials than those who have to switch from high-similarity to lowsimilarity comparisons. However, if progressive alignment holds here, then children who first receive high-similarity comparisons will perform better on the subsequent low-similarity comparisons than will children who receive low-similarity comparisons throughout.

\section{Method}

Participants. A total of 963- and 4-year-olds participated individually in our laboratory. The 3-year-olds ranged in age from 40 to 48 months $(M=44)$, and the 4-year-olds ranged in age from 52 to 60 months $(M=56)$. There were 25 children 
of each age in the progressive alignment condition, and 23 of each age in the control condition. There were roughly the same number of boys and girls in each age and condition.

Materials. We used six of the eight groups of animals from Experiment 2 (omitting the groups with the hair and eyebrow parts) and constructed additional items such that each standard had two test sets rather than one test set as in Experiments 1 and 2. (Recall that each set consists of two trials.) The first test set differed by condition: the progressive alignment group received a high-similarity set and the control group received a low-similarity set. The second test set was a low-similarity set for both conditions. Within each set, on the first trial the incorrect variant simply lacked the key part (as in Experiment 1); on the second trial, the incorrect variant had an extraneous non-matching part, (as in Experiment 2). Figure 5 shows a sample group shown in the progressive alignment condition and the corresponding group shown in the control condition.

The pretest items were the same as in the previous experiments. In addition, we inserted three catch trials (one each after the third, fourth and fifth sets) to check whether children were maintaining attention to the task despite receiving four pairs of variants per standard. Like the pre-test items, the catch trial items were designed to be very easy, by having large parts whose absence significantly changed the external shape of the objects; they were a mug (the part was a handle), a horse (sad-
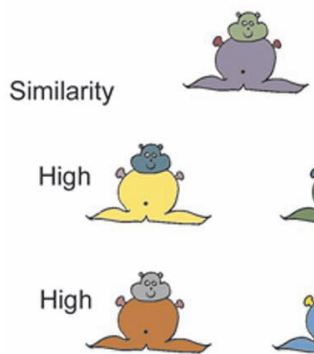

Low

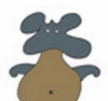

Low

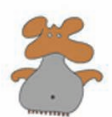

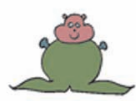
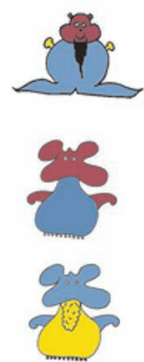
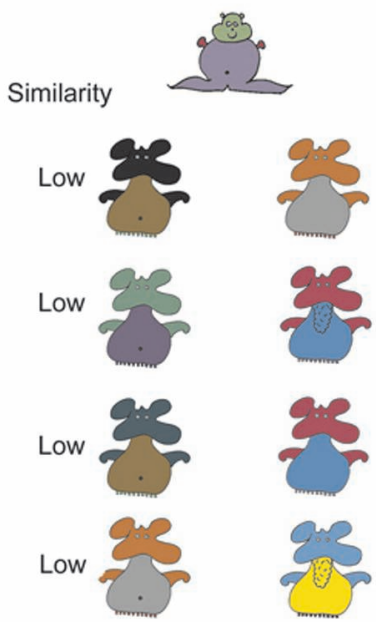

FIGURE 5 Sample progressive alignment sets and Control sets for Experiment 3. 
dle), and a house (roof). Children performed nearly flawlessly (96\% correct) on these trials.

Procedure. Participants were seated at a table across from the experimenter. The children were introduced to Jo-Jo the seal. They were told that "Jo-Jo is a very special seal because he has his own language," and they were instructed that they would learn some words in Jo-Jo's language. All the children were shown the pre-test items, and if they did not choose the matching part, the experimenter corrected them and directed their attention to the part. (As in the previous studies, no feedback was given during the experimental trials and the catch trials.) The children were shown a standard and told that "Jo-Jo says this one has a blick." The experimenter then laid down the first pair of test variants and said, "Which one of these also has a blick?" This was repeated for the remaining three pairs. The catch trials were presented in the same manner as the test sets, except that the catch trials had only one pair of test variants.

In the first set for each standard, in the progressive alignment condition, the two pairs of test variants were similar to the standard; in the control condition, the test pairs were dissimilar to the standard. The second set was the same for all children: two pairs of test variants that were dissimilar to the standard. This was a switch for the children in the progressive alignment condition, but a continuation of two more trials of the same kind for those in the control condition.

Design and scoring. All children saw 6 standards, each with 2 sets, for a total of 12 sets (with each set containing two trials). As before, the primary dependent measure was the number correct on each set. These data were analyzed in two parallel between-subject ANOVAs (one for each set) with the design 2 (Age: 3, 4) $\times 2$ (Condition: Progressive Alignment, Control). In addition, given our particular interest in examining how children's performance on the first set was related to their performance on the second set, we also ran a binary logistic regression model using children's first set performance to predict their second set performance.

\section{Results and Discussion}

To presage the quantitative results, there were two main findings (Figure 6). First, the pattern of results replicated the similarity effect found in the earlier studies: Children performed better on high-similarity trials than low-similarity trials. More importantly, we found the predicted effect of progressive alignment: Children who received high-similarity trials in the first set showed elevated performance on the second (low- similarity) set, relative to the control group children who had received low-similarity trials in the first set. Because the second set of trials was identical for the two groups of children, the elevated performance of the progressive alignment group can be attributed to their prior experience with high- 
similarity, easily alignable trials. Such a transfer effect suggests that structural alignment not only can support immediate part identification but also can confer insight that potentiates future extensions.

First set performance. The pattern of results replicated the similarity effect found in the earlier studies. Children in the progressive alignment condition (who received high-similarity trials) performed better than children in the control condition (who received low-similarity trials), .54 versus $.36, F(1,92)=12.28, p<.005$, $M S E=.07, \eta_{\mathrm{p}}{ }^{2}=.12$. As expected, the 4-year-olds (.55) performed better than the 3 -year-olds $(.34), F(1,92)=14.29, p<.001, \eta_{\mathrm{p}}{ }^{2}=.13$. The two factors did not interact. The similarity advantage was reliable for 4-year-olds, $F(1,92)=9.32, p<$ $.005, \eta_{\mathrm{p}}^{2}=.09$, but not for 3-year-olds, $F(1,92)=3.63, p=.06$. However, 3 -year-olds in the progressive alignment condition (with high-similarity matches) performed above chance, $t(24)=2.86, p<.01$, whereas those in the control condition (who had low-similarity matches) did not. The number of children performing above chance (at least 4 of 6 sets correct) was greater in the progressive alignment (high-similarity) condition than in the control condition: for the 3-year-olds, .32 vs. .04, Fisher's exact test, $p<.05$; and for the 4 -year-olds, $68 \%$ vs. $26 \%, \chi^{2}(1, N=$ $48)=8.43, p<.005$.

Second set performance. In the second set, both groups received the same items. Nevertheless, the progressive alignment group performed far better than the

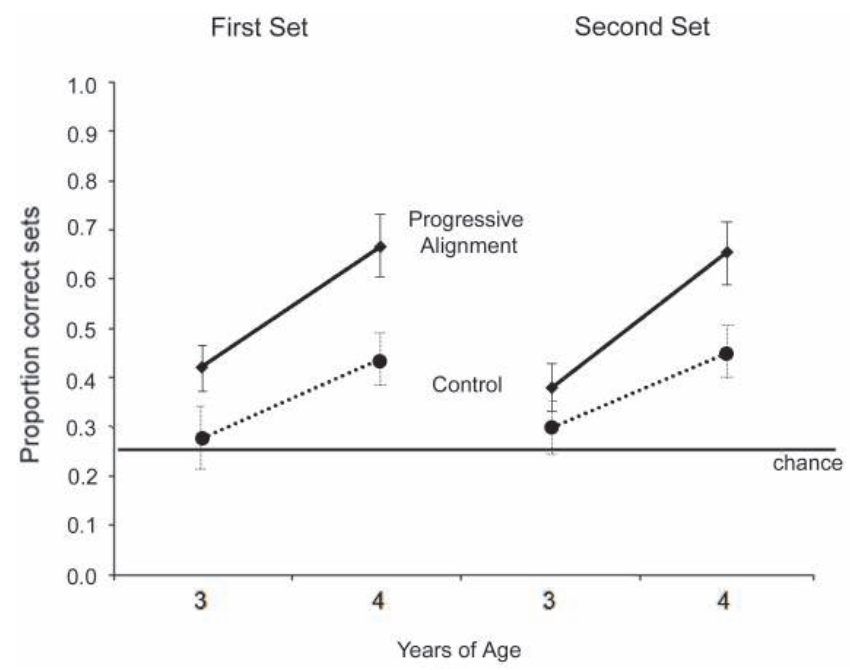

FIGURE 6 Performance in Experiment 3 by set for the Progressive Alignment Condition (the first set used high-similarity items, the second set used low-similarity items) and the Control Condition (both sets used low-similarity items). 
control group, confirming the prediction that close similarity comparisons potentiate analogical matches. Indeed, for these children, performance on the second set (with low-similarity trials) was much like performance on the first set (with high-similarity trials). A mixed measures ANOVA with set number as a repeated measure showed no effects due to set. Children in the progressive alignment condition (.51) performed better those in the control condition (.37), $F(1,92)=7.05, p<$ $.01, M S E=.07, \eta_{\mathrm{p}}^{2}=.07$. There was also an age effect: 4-year-olds (.55) performed better than 3-year-olds $(.33), F(1,92)=15.55, p<.001, \eta_{\mathrm{p}}{ }^{2}=.15$. The progressive alignment advantage was reliable for 4-year-olds, $F(1,92)=7.20, p<.01$, $\eta_{\mathrm{p}}{ }^{2}=.07$, but not for 3-year-olds, $F=1.15, p=.29$. However, the 3 -year-olds in the progressive alignment condition did perform above the .25 chance level, $t(24)=$ $2.53, p<.05$. Across all three studies, this was the only group of 3-year-olds who performed above chance on low-similarity items.

Our theoretical account is that high similarity facilitates structural alignment, allowing children to notice correspondences across items and to notice the matching part that the contrasting test item does not share. For this reason, we examined the likelihood that children would perform correctly on the second set of items given their performance on the first set of items. If children were unsuccessful on the first set, they were rarely successful on the second, both for the progressive alignment condition ( $p$ [set success $_{2} \mid$ set $_{1}$ failure] $=.25$ ) and the control condition ( $p$ [set success $_{2} \mid$ set $_{1}$ failure] = .28). In contrast, the conditions differed substantially if there was initial success. For the progressive alignment group, success bred success $\left(p\right.$ (set success $_{2}$ set $_{1}$ success $\left.)=.74\right)$; but this did not hold for the con-

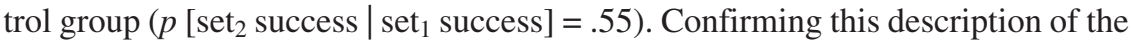
conditional probabilities, a binary logistic regression showed that set ${ }_{1}$ performance was a reliable predictor of $\operatorname{set}_{2}$ performance $\left(\mathrm{B}=1.23, \mathrm{SE}=.24\right.$, Wald $\chi^{2}=$ $26.05, p<.001)$, and that this effect was moderated by a reliable interaction effect with condition $\left(\mathrm{B}=.85, \mathrm{SE}=.27\right.$, Wald $\left.\chi^{2}=9.67, p<.005\right)$. This means that being correct on a given high-similarity set was a better predictor of being correct on the corresponding low-similarity set for the progressive alignment group than it was for the control group. This is all the more striking in view of the fact that for the control group, the second set was essentially the same as the first. This result is consistent with our claim that comparing closely similar items renders the common relational structure more salient, and that this potentiates processing more distant matches based on the same structure.

\section{GENERAL DISCUSSION}

Across three experiments, we found evidence that comparison processes can help young children learn part terms. First, at least by four years of age, children can compare and align pairs of exemplars and use that alignment to notice a matching 
part. Second, the results bore out the "relational shift" prediction that children would find high-similarity pairs easier to align than low-similarity pairs. Across all three studies, children were more accurate in identifying which variant had the matching body part if the variants were similar to the standard than if they were not. Third, children can learn part structure via the progressive alignment process. In Experiment 3, children who first received high-similarity variants were subsequently better at identifying the part in low-similarity items than the control group children, who had twice as much the exposure to low-similarity items. In other words, aligning pairs that are highly similar facilitated aligning new pairs that were quite dissimilar, but that shared relational structure.

The part-name task was challenging in several ways. The part was never explicitly pointed out, even in the initial standard; the children were simply asked, "This one has a dax. Which one of these has a dax?" Further, all the figures were novel animals or objects, so children could not rely on knowledge of familiar categories. Because the figures were not themselves named, children could not use mutual exclusivity to buttress their understanding that the part word referred to a part, and not the whole. Finally, in Experiment 2 and half the trials in Experiment 3, the incorrect variant (that lacked the corresponding part) had a distinctive part of its own. Thus children could not solve the task by choosing the variant with an extra part; they had to choose the one whose part corresponded to one in the standard. Given only the part-whole phrasing "x has a y" and the opportunity to align the standard with each of the alternatives, they were able to identify the part in question. Such a process could allow children to learn something about part structure from ordinary experience, perhaps even from overheard conversations.

These results fit with the predictions of structure-mapping theory (Gentner, 1983; Gentner \& Markman, 1997), which states that comparison is a process of structural alignment and mapping that promotes the salience of common relational systems and alignable differences Markman \& Gentner, 1999; (Gentner \& Markman, 1997). When children succeed in aligning the representations of the standard and the variant, the overall structural alignment supports noticing the correspondence between the key parts. However, because young children's relational representations tend to be fragile, they are more likely to arrive at the correct alignment if there are surface similarities to guide the mapping; hence, performance is better with high-similarity than with low-similarity pairs.

Perhaps the most surprising result is the gain in performance on low-similarity pairs following high-similarity pairs. One might have expected the opposite: that experience with high-similarity comparisons would fail to transfer beyond a close similarity radius. However, this finding that close comparisons potentiate far comparisons is consistent with prior research showing that a close literal similarity comparison, though it may seem obvious to adults, can be informative to children. We have repeatedly found that very close similarity comparisons can promote relational insight in young children, and that this insight can potentiate transfer to new 
exemplars that share relational structure but are dissimilar on the surface (Kotovsky \& Gentner, 1996; Gentner \& Namy, 1999; Loewenstein \& Gentner, 2001; Namy \& Gentner, 2002).

This finding adds to evidence that comparison promotes the salience of connected relational systems over other commonalities (Clement \& Gentner, 1991; Gentner \& Markman, 1997). When two representations (even literally similar ones) are aligned, the maximal common relational system is preferentially highlighted and becomes more available for subsequent processing. Thus, in the current study, children who began with high-similarity pairs gained relational insight that they then applied to the low-similarity pairs. The surface commonalities in the initial pairs helped children achieve the correct alignment, but once this alignment was achieved, the previously implicit common relational structure became more salient.

There is prior evidence for the claim that comparison highlights structural commonalities. For example, Gentner \& Namy (1992; Namy and Gentner, 2002) investigated the role of comparison in novel noun extension. Four-year-old children were given either one standard (e.g., an apple) or two highly similar standards from the same superordinate category (e.g., an apple and an orange). They were given a name for the standard(s) (e.g., "blicket") and asked to choose another blicket from two alternatives: a perceptually similar object from a different taxonomic category (e.g., a balloon) and a perceptually dissimilar object from the same category (e.g., a banana). Children who received only one standard mostly chose the surfacesimilar alternative; but those who compared two standards were able to reject the perceptually similar lure in favor of the relationally similar item. Because the two standards shared close perceptual similarity as well as relational similarity, Gentner and Namy concluded that the process of comparing the standards had preferentially highlighted the relational commonalities.

The effect of alignment in promoting learning is evident across a wide span of ages and cognitive tasks. For example, Oakes (2001) found that 4- to 6-month-old infants more readily formed perceptual categories such as dog (and discriminated dogs from perceptually similar cats) when the infants were first given the opportunity to view and compare pairs of objects than when the objects were presented one at a time. Loewenstein and Gentner (2001) found benefits of close comparison on preschool children's ability to perform a spatial mapping task. As in the classic DeLoache (1989) studies, children searched for a hidden object in a new room, having been told that it was "in the same place" as an object they had seen hidden in an initial room. The new room had the same spatial configuration as the initial room, but differed in surface details such as the shape and color of the furniture. Consistent with DeLoache's findings, 3- to 4-year-olds found this task rather challenging. The key manipulation was that prior to the task, half the children compared the initial room with another room that was identical, except for color. Children who engaged in this high-similarity comparison task performed better on the subsequent mapping task than those who viewed the first two rooms separately, 
again suggesting that the initial comparison rendered the common spatial relational structure more salient and thus helped children to align the initial model with the dissimilar model at test.

In addition to comparison, children also appear to use category knowledge, often as provided by linguistic labels, to identify parts. Although we did not provide category labels, we suspect doing so would also have yielded an advantage, based on Saylor, et al.'s (2002) work and by analogy with findings on children's acquisition of property terms (Hall, Waxman \& Hurwitz, 1993; Markman \& Wachtel, 1988). Further, comparison processes and category knowledge can be usefully combined. Mintz and Gleitman (2002) found that 2- and 3-year-old children could more easily learn new property names if they modified a familiar basic-level category label (e.g., "That's a drin car! And that's a drin ball"). Children were given an opportunity to draw comparisons across items, but these comparisons were more effective when children heard basic-level category labels than when they heard only generic terms (e.g., "That's a drin thing! And here's another drin one!"). We conjecture that the basic level terms helped children to form richer representations which then informed the comparison process.

The above studies underline the importance of language in guiding children's processing. But children can sometimes learn via comparison even with minimal linguistic support. Mintz (2005), using the property-naming paradigm just discussed, found that children could successfully map new adjectives to properties modifying superordinate-level category labels, provided the pairs of objects were familiar. And Waxman and Klibanoff (2000) found that preschool children who first learned a novel adjective across similar items from the same basic-level category were subsequently better than a baseline group at applying the property name to a dissimilar item from a different basic-level category (with the same superordinate). This suggests that a common mechanism-a progressive alignment-may account for the unsupervised learning of both property and part label acquisition.

Children learn word meanings at a prodigious rate. Some of this learning is supported by caretakers who explicitly explain the meanings of new words, but much of it must be accomplished with less direct guidance. Our findings here and in prior work suggest that structural alignment and mapping processes are integral to children's learning of word meanings-that words act as "invitations to compare" (Gentner, 2003; Gentner \& Loewenstein, 2002; Gentner \& Namy, 1999). Indeed, Gentner and Namy (2006) have proposed that structural alignment processes contribute to language learning more broadly. In the present paper, our studies provide evidence that structure-mapping processes can help children work out the referents of part terms, even in the absence of category information.

Our evidence here and elsewhere indicates that high-similarity comparisons can facilitate subsequent low-similarity comparisons. This is important because highly similar pairs are likely to be spontaneously noticed and compared, even by young children. If, as our findings suggest, these high-similarity comparisons po- 
tentiate further, less obvious comparisons, then children's natural patterns of attention and curiosity can lead them to deeper knowledge of a domain's relational structure. We suggest that progressive alignment provides a bootstrapping mechanism that enables children to gain insight from experience.

\section{ACKNOWLEDGEMENTS}

This research was supported by NSF-ROLE award 21002/REC-0087516 and by NSF SLC Grant SBE-0541957, the Spatial Intelligence and Learning Center (SILC). We thank members of the Cognition and Language Lab at Northwestern University for discussions of the ideas, and Kathleen Braun for technical assistance.

\section{REFERENCES}

Akhtar, N. (2005). The robustness of learning through overhearing. Developmental Science, 8, 199-209.

Akhtar, N., Jipson, J., \& Callanan, M. A. (2001). Learning words through overhearing. Child Development, 72, 416-430.

Akhtar, N., \& Montague, L. (1999). Early lexical acquisition: The role of cross-situational learning. First Language, 19, 347-358.

Clark, E. V. (1993). The lexicon in acquisition. Cambridge: Cambridge University Press.

Clement, C. A., \& Gentner, D. (1991). . Cognitive Science, 15, 89-132.

DeLoache, J. S. (1989). Young children's understanding of the correspondence between a scale model and a larger space. Cognitive Development, 4, 121-139.

Falkenhainer, B., Forbus, K. D., \& Gentner, D. (1989). The structure-mapping engine: Algorithm and examples. Artificial Intelligence, 41, 1-63.

Gentner, D. (1983). Structure-mapping: A theoretical framework for analogy. Cognitive Science, 7, $155-170$.

Gentner, D. (1988). Metaphor as structure mapping: The relational shift. Child Development, 59, 47-59.

Gentner, D. (2003). Why we're so smart. In D. Gentner and S. Goldin-Meadow (Eds.), Language in mind: Advances in the study of language and cognition (pp. 195-235). Cambridge, MA: MIT Press. Gentner, D, \& Loewenstein, J. (2002). Relational language and relational thought. In J. Byrnes \& E. Amsel (Eds.), Language, literacy, and cognitive development (pp. 87-120). Mahwah, NJ: Erlbaum.

Gentner, D., Loewenstein, J., \& Thompson, L. (2003). Learning and transfer: A general role for analogical encoding. Journal of Educational Psychology, 95(2), 393-408.

Gentner, D., \& Markman, A. B. (1997). Structure mapping in analogy and similarity. American Psychologist, 52, 45-56.

Gentner, D., \& Medina, J. (1998). Similarity and the development of rules. Cognition, 65, 263-297.

Gentner, D., \& Namy, L. L. (1999). Comparison in the development of categories. Cognitive Development, 14, 487-513.

Gentner, D., \& Namy, L. L. (2006). Analogical processes in language learning. Current Directions in Psychological Science, 15, 297-301.

Gentner, D., \& Rattermann, M. J. (1991). Language and the career of similarity. In S. A. Gelman \& J. P. Brynes (Eds.), Perspectives on thought and language: Interrelations in development (pp. 225-277). London: Cambridge University Press. 
Hall, D. G., Waxman, S. R., \& Hurwitz, W. M. (1993). . Child Development, 64, 1661-1664.

Klibanoff, R. S., \& Waxman, S. R. (2000). Basic level object categories support the acquisition of novel adjectives: Evidence from preschool-aged children. Child Development, 71, 649-659.

Kobayashi, H. (1997). The role of actions in making inferences about the shape and material of solid objects among Japanese 2-year-old children. Cognition, 3, 251-269.

Kobayashi, H. (1998). How 2-year-olds learn novel part names of unfamiliar objects. Cognition, 68, B41-B51.

Kotovsky, L., \& Gentner, D. (1996). Comparison and categorization in the development of relational similarity. Child Development, 67, 2797-2822.

Kurtz, K. J., Miao, C., \& Gentner, D. (2001). Learning by analogical bootstrapping. Journal of the Learning Sciences, 10, 417-446.

Loewenstein, J., \& Gentner, D. (2001). Spatial mapping in preschoolers: Close comparisons facilitate far mappings. Journal of Cognition and Development, 2, 189-219.

Loewenstein, J., \& Gentner, D. (2005). Relational language and the development of relational mapping. Cognitive Psychology, 50, 315-353.

Loewenstein, J., Thompson, L., \& Gentner, D. (1999). Analogical encoding facilitates knowledge transfer in negotiation. Psychonomic Bulletin \& Review, 6, 586-597.

Markman, A. B., \& Gentner, D. (1993). Structural alignment during similarity comparisons. Cognitive Psychology, 25, 431-467.

Markman, E. M., \& Wachtel, G. F. (1988). Children's use of mutual exclusivity to constrain the meanings of words. Cognitive Psychology, 20, 121-157.

Markman, E. M. (1989). Categorization and naming in children: Problems of induction. Cambridge, MA: MIT Press.

Masur, E. F. (1997). Maternal labeling of novel and familiar objects: Implications for children's development of lexical constraints. Journal of Child Language, 24, 427-439. Cambridge University Press, US

Mintz, T. H. (2005). Linguistic and conceptual influences on adjective acquisition in 24- and 36-month-olds. Developmental Psychology, 41, 17-29.

Mintz, T. H., \& Gleitman, L. R. (2002) Adjectives really do modify nouns: The incremental and restricted nature of early adjective acquisition. Cognition, 84, 267-293.

Namy, L. L., \& Gentner, D. (2002). Making a silk purse out of two sow's ears: Young children's use of comparison in category learning. Journal of Experimental Psychology: General, 131, 5-15.

Oakes, L. M. (2001). The role of comparison in category formation in infancy. Paper presented at the 68th Anniversary Meeting of the Society for Research in Child Development, Minneapolis, MN.

Rattermann, M. J., \& Gentner, D. (1998). More evidence for a relational shift in the development of analogy: Children's performance on a causal-mapping task. Cognitive Development, 13, 453-478.

Rosch, E., Mervis, C. B., Gray, W., Johnson, D., \& Boyes-Braem, P. (1976). Basic objects in natural categories. Cognitive Psychology, 8, 382-439.

Saylor, M. M., \& Sabbagh, M. A. (2004). Different kinds of information affect word learning in the preschool years: The case of part-term learning. Child Development, 75, 395-408.

Saylor, M. M., Sabbagh, M. A., \& Baldwin, D. A. (2002). Children use whole-part juxtaposition as a pragmatic cue to word meaning. Developmental Psychology, 38, 993-1003.

Tversky, B. (1989). Parts, partonomies, and taxonomies. Developmental Psychology, 25, 983-995.

Tversky, B., \& Hemenway, K. (1984). Objects, parts, and categories. Journal of Experimental Psychology: General, 113, 169-193.

Waxman, S. R., \& Klibanoff, R. S. (2000). The role of comparison in the extension of novel adjectives. Developmental Psychology, 36, 571-581.

Wolff, P., \& Gentner, D. (2000). Evidence for role-neutral initial processing of metaphors. Journal of Experimental Psychology: Learning, Memory, and Cognition, 26, 529-541. 